Original Paper (Invited)

\title{
A New Approach in Numerical Assessment of the Cavitation Behaviour of Centrifugal Pumps
}

\author{
Adrian Stuparu ${ }^{1}$, Romeo Susan-Resiga ${ }^{1}$, Liviu Eugen Anton ${ }^{1}$ and Sebastian Muntean ${ }^{2}$ \\ ${ }^{1}$ Department of Hydraulic Machinery, Politehnica University of Timisoara \\ Bd. Mihai Viteazu 1, Timisoara, 300222, Romania, \\ astuparu@mh.mec.upt, resiga@mh.mec.upt.ro, liviu.anton@mec.upt.ro \\ ${ }^{2}$ Centre of Advanced Research in Engineering Science, Romanian Academy-Timisoara Branch, \\ Bd. Mihai Viteazu 24, Timisoara, 300223, Romania, seby@acad-tim.tm.edu.ro
}

\begin{abstract}
The paper presents a new method for the analysis of the cavitation behaviour of hydraulic turbomachines. This new method allows determining the coefficient of the cavitation inception and the cavitation sensitivity of the turbomachines. We apply this method to study the cavitation behaviour of a large storage pump. By plotting in semi-logarithmic coordinates the vapour volume versus the cavitation coefficient, we show that all numerical data collapse in an exponential manner. By analysis of the slope of the curve describing the evolution of the vapour volume against the cavitation coefficient we determine the cavitation sensitivity of the pump for each operating point.
\end{abstract}

Keywords: cavitation inception, cavitation sensibility, pumping head drop, storage pump

\section{Introduction}

In many engineering applications, cavitation has been the subject of extensive theoretical and experimental research since it has predominantly been perceived as an undesirable phenomenon. This is mainly due to the detrimental effects of cavitation such as erosion, noise and vibrations, caused by the growth and collapse of vapour bubbles. The ability to model cavitating flows has drawn strong interest in CFD community. It covers a wide range of applications, such as pumps, hydraulic turbines, inducers and fuel cavitation in orifices as commonly encountered in fuel injection systems. Fluid machinery is a common application where low pressures are routinely generated by the machine action, e.g. on blade surfaces, with a consequent possibility of cavitation. Existence of cavitation is often undesired, because it can degrade the device performance, produce undesirable noise, lead to physical damage to the device and affect the structural integrity. Details of the existence, extent and effects of cavitation can be of significant help during the design stages of fluid machinery, in order to minimize cavitation or to account for its effects and optimize the design.

Past several decades have seen considerable research on cavitation and extensive reviews are available in the literature [1], [3]. Different aspects of this complex phenomenon have been explored, including, e.g., cavitation bubble collapse and erosion damage, cavitation acoustics, cloud cavitation and rotating cavitation.

Viscous flow models, which regard the cavitating flow as the bubbly flow containing spherical bubbles, were introduced to provide highly accurate calculations. In the viscous flow models, the Navier-Stokes equation including cavitation bubble is solved in conjunction with Rayleigh's equation governing the change in the bubble radius. To account for the cavitation dynamics in a more flexible manner a transport equation model has been developed. In this approach volume or mass fraction of liquid (and vapour) phase is convective. Singhal et al. [5] have employed similar models based on this concept with differences in the source terms.

Hirschi et al. [8] proposed a method, which allows the performance drop prediction, consisting of assuming the cavity interface as a free surface boundary of the computation domain and that leads to compute only a single phase flow. The unknown shape of the interface is determined using an iterative procedure matching the cavity surface to a constant pressure boundary. The numerical results were in good agreement with the measurements. The originality of the method was that the adaptation process is done apart from the flow calculation, allowing the use of any numerical simulation software. Ait-Bouziad [11] used the mixture model for the case study of an industrial inducer and found that this model provides satisfactory results for the prediction of the cavitation flow behaviour and performance drop estimation. Pouffary et al. [9] investigate the cavitating flow in turbomachinery

Accepted for publication December 19, 2010: Paper number O10055S

Corresponding author: Adrian Stuparu, PhD Assistant Professor, astuparu@mh.mec.upt.ro

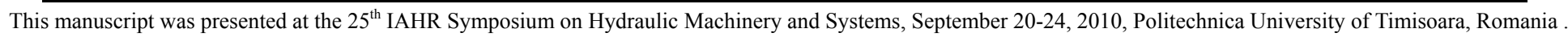


with the help of numerical simulation using a barotropic state law to model cavitation phenomenon. From the comparison of the numerical results with experiment a good agreement results regarding the head drop of the investigated turbomachines. Flores et al. [10] used a numerical model based on homogeneous approach of the multiphase flow coupled with a barotropic state law for the cool water vapour-liquid mixture. The numerical results showed a good prediction of the head drop and were compared with experimental results leading to a good overlapping.

This paper presents the computational analysis of the cavitational behaviour of a storage pump for five operating points by using the new method for determining the cavitation incipience coefficient and the curves describing the cavitation sensitivity. Two-phase cavitating flow models based on homogeneous mixture approach, with a transport equation for the vapour volume fraction have been included in expert commercial codes such as FLUENT [6] that we use. We conclude that, for steady cavitating flow, the model presented in this paper, captures correctly the vapour phase distribution on the blade of the pump impeller. The current effort is based on the application of the full cavitation model that utilizes the modified Rayleigh-Plesset equations for bubble dynamics and includes the effects of turbulent pressure fluctuations and non-condensable gases (ventilated cavitation) to rotating cavitation in different types of fluid turbomachines.

\section{Numerical method of modelling cavitating flow}

The cavitation model implemented in FLUENT, [6], is based on the so-called "full cavitation model," developed by Singhal et al. [5] and it accounts for all first-order effects like phase change, bubble dynamics, turbulent pressure fluctuations and noncondensable gases. Unlike the original approach assuming single-phase, isothermal, variable fluid density flows, the cavitation model in FLUENT is under the framework of multiphase flows. It has the capability to account for two phases flows, the effects of slip velocities between the liquid and gaseous phases, and the thermal effects and compressibility of both liquid and gas phases. The cavitation is modelled in this paper with the help of mixture multiphase model from FLUENT.

The mixture model solves the continuity equation for the mixture, the momentum equation for the mixture, the volume fraction equation for the secondary phase, vapour mass fraction and vapour transport equation, as well as algebraic expressions for the relative velocities.

The continuity equation for the mixture is

$$
\frac{\partial}{\partial t}\left(\rho_{m}\right)+\nabla \cdot\left(\rho_{m} \vec{v}_{m}\right)=0
$$

where $\vec{v}_{m}$ is the mass-averaged velocity given by:

$$
\vec{v}_{m}=\frac{\alpha_{L} \rho_{L} \vec{v}_{L}+\alpha_{V} \rho_{V} \vec{v}_{V}}{\rho_{m}}
$$

and $\rho_{m}$ is the mixture density:

$$
\rho_{m}=\alpha_{L} \rho_{L}+\alpha_{V} \rho_{V}
$$

$\alpha_{L}$ and $\alpha_{V}$ is the volume fraction of liquid and vapour phase:

$$
\alpha_{L}+\alpha_{V}=1
$$

The momentum equation for the mixture can be obtained by summing the individual momentum equations for all phases and it is expressed as:

$$
\frac{\partial}{\partial t}\left(\rho_{m} \vec{v}_{m}\right)+\nabla \cdot\left(\rho_{m} \vec{v}_{m} \vec{v}_{m}\right)=-\nabla p_{m}+\nabla \cdot\left[\mu_{m}\left(\nabla \vec{v}_{m}\right)+\nabla \vec{v}_{m}^{T}\right]+\rho_{m} \vec{g}+\vec{F}+\nabla \cdot\left(\sum_{k=1}^{2} \alpha_{k} \rho_{k} \vec{v}_{d r, k} \vec{v}_{d r, k}\right)
$$

where $\vec{F}$ is a body force and $\mu_{m}$ is the viscosity of the mixture:

$\vec{v}_{d r, V}$ is the drift velocity for the vapour phase:

$$
\mu_{m}=\alpha_{L} \mu_{L}+\alpha_{V} \mu_{V}
$$

$$
\vec{v}_{d r, V}=\vec{v}_{V}-\vec{v}_{m}
$$

The relative velocity (also referred to as the slip velocity) is defined as the velocity of the vapour phase relative to the velocity of the liquid phase:

$$
\vec{v}_{V L}=\vec{v}_{V}-\vec{v}_{L}
$$

The mass fraction for the two phases is defined as

$$
\begin{aligned}
& f_{L}=\frac{\alpha_{L} \rho_{L}}{\rho_{m}} \\
& f_{V}=\frac{\alpha_{V} \rho_{V}}{\rho_{m}}
\end{aligned}
$$

The drift velocity and the relative velocity are connected by the following expression:

$$
\vec{v}_{d r, V}=\vec{v}_{V L}-\left(f_{L} \vec{v}_{L L}+f_{V} \vec{v}_{L V}\right)
$$

The mixture model implemented in FLUENT makes use of an algebraic slip formulation. The basic assumption of the algebraic slip mixture model is that to prescribe an algebraic relation for the relative velocity, a local equilibrium between the phases should be reached over short spatial length scale. Following Manninen et al. [3], the form of the relative velocity is given by: 


$$
\vec{v}_{V L}=\frac{\tau_{V}}{c_{d r a g}} \frac{\left(\rho_{V}-\rho_{m}\right)}{\rho_{V}} \vec{a}
$$

where $\tau_{V}$ is the bubble relaxation time:

$$
\tau_{V}=\frac{\rho_{V} d_{V}^{2}}{18 \mu_{L}}
$$

$d_{V}$ is the diameter of the bubbles for the vapour phase, $\vec{a}$ is the vapour bubble's acceleration. The default drag function $c_{d r a g}$ is taken from Schiller and Naumann [12]:

and the acceleration is of the form:

$$
c_{\text {drag }}= \begin{cases}1+0.15 \mathrm{Re}^{0.687} & \mathrm{Re} \leq 1000 \\ 0.0183 \mathrm{Re} & \mathrm{Re}>1000\end{cases}
$$

$$
\vec{a}=\vec{g}-\left(\vec{v}_{m} \cdot \nabla\right) \vec{v}_{m}-\frac{\partial \vec{v}_{m}}{\partial t}
$$

The simplest algebraic slip formulation is the so-called drift flux model, in which the acceleration of the bubble is given by gravity and/or a centrifugal force and the bubble relaxation time is modified to take into account the presence of other particles.

In turbulent flows the relative velocity should contain a diffusion term due to the dispersion appearing in the momentum equation for the dispersed phase. FLUENT adds this dispersion to the relative velocity:

$$
\vec{v}_{V L}=\frac{\left(\rho_{V}-\rho_{m}\right) d_{V}^{2}}{18 \mu_{L} c_{\text {drag }}} \vec{a}-\frac{v_{m}}{\alpha_{V} \sigma_{D}} \nabla \alpha_{L}
$$

where $v_{m}$ is the mixture turbulent viscosity and $\sigma_{D}$ is a Prandtl dispersion coefficient.

From the continuity equation for vapour phase, the volume fraction equation for the vapour phase can be obtained:

$$
\frac{\partial}{\partial t}\left(\alpha_{V} \rho_{V}\right)+\nabla \cdot\left(\alpha_{V} \rho_{V} \vec{v}_{m}\right)=-\nabla \cdot\left(\alpha_{V} \rho_{V} \vec{v}_{d r, V}\right)+\left(\dot{m}_{L V}-\dot{m}_{V L}\right)
$$

A vapour transport equation governs the vapour mass fraction, $f_{V}$, given by

$$
\frac{\partial}{\partial t}\left(\rho_{m} f_{V}\right)+\nabla\left(\rho_{m} \vec{v}_{V} f_{V}\right)=\nabla\left(\gamma \nabla f_{V}\right)+R_{e}-R_{c}
$$

where $\gamma$ is the effective exchange coefficient, and $R_{e}$ and $R_{c}$ are the vapour generation and condensation rate terms (or phase change rates). The rate expressions are derived from Rayleigh-Plesset equations, and limiting bubble size considerations (interface surface area per unit volume of vapour), [5]. These rates are functions of the instantaneous, local static pressure and are given by:

$$
\begin{gathered}
R_{e}=C_{e} \frac{v_{c h}}{\sigma_{t}} \rho_{L} \rho_{V} \sqrt{\frac{2\left(p_{s a t}-p\right)}{3 \rho_{L}}}\left(1-f_{V}\right), \text { for } p<p_{s a t} \\
R_{c}=C_{c} \frac{V_{c h}}{\sigma_{t}} \rho_{L} \rho_{V} \sqrt{\frac{2\left(p-p_{s a t}\right)}{3 \rho_{L}}} f_{V}, \text { for } p>p_{\text {sat }}
\end{gathered}
$$

where $\sigma_{t}$ is the surface tension coefficient of the liquid, $p_{\text {sat }}$ is the liquid saturation vapour pressure at the given temperature, $C_{e}$ and $C_{c}$ are empirical constants with the default values $C_{e}=0.02$ and $C_{c}=0.01$ and $v_{c h}$ is a characteristic velocity, which is approximated by the local turbulence intensity:

$$
v_{c h}=\sqrt{k}
$$

The cavitation model implemented in FLUENT take into account the pressure fluctuation induced by turbulence by raising the phase-change threshold pressure from $p_{\text {sat }}$ to:

where $k$ is the local turbulence kinetic energy.

$$
\begin{gathered}
p_{\text {vap }}=\frac{p_{\text {sat }}+p_{\text {turb }}}{2} \\
p_{\text {turb }}=0.39 \rho_{m} k
\end{gathered}
$$

This cavitation model assumes that the working fluid is a mixture of liquid and gaseous phase, with the gaseous phase comprising of the liquid vapour and the noncondensable gases. The density of the mixture is calculated as:

$$
\rho_{m}=\alpha_{V} \rho_{V}+\alpha_{g} \rho_{g}+\left(1-\alpha_{V}-\alpha_{g}\right) \rho_{L}
$$

where $\rho_{L}, \rho_{V}$ and $\rho_{g}$ are the densities of the liquid, the vapour, and the noncondensable gases, respectively, and $\alpha_{L}, \alpha_{V}$ and $\alpha_{g}$ are the respective volume fractions. The connection between the mass fraction in Eq. 18, Eq.19 and Eq. 20 and the volume fraction in Eq. 24 is:

$$
\alpha_{V}=f_{V} \frac{\rho_{m}}{\rho_{V}}
$$

The combined volume fraction of vapour and gas is commonly referred to as the void fraction. It can be observed that the noncondensable gas is not defined as a phase or a material. When using the ideal gas law to compute the noncondensable gas density, the molecular weight and temperature is required. By default, the gas is assumed to be air and the molecular weight is set 
to 29 . However, if the noncondensable gas is not air, then the molecular weight can be changed by using a text command.

After accounting for the effect of turbulence-induced pressure fluctuations and noncondensable gases, the final phase rate expressions are written as:

$$
\begin{gathered}
R_{e}=C_{e} \frac{\sqrt{k}}{\sigma_{t}} \rho_{L} \rho_{V} \sqrt{\frac{2\left(p_{\text {vap }}-p\right)}{3 \rho_{L}}}\left(1-f_{V}-f_{g}\right), \text { for } p<p_{\text {vap }} \\
R_{c}=C_{c} \frac{\sqrt{k}}{\sigma_{t}} \rho_{L} \rho_{V} \sqrt{\frac{2\left(p-p_{\text {vap }}\right)}{3 \rho_{L}}} f_{V}, \text { for } p>p_{\text {vap }}
\end{gathered}
$$

In the standard two-phase cavitation model implemented in FLUENT the following assumptions are made:

- the system under investigation involves only two phases (a liquid and its vapour), and a certain fraction of separately modelled noncondensable gases

- both bubble formation (evaporation) and collapse ( condensation) are taken into account in the model

- the mass fraction of noncondensable gases is known in advance

- the cavitation model accounts for the mass transfer between a single liquid and its vapour

The FLUENT code requires the following methodology for computing cavitating flows. First, a steady solution is obtained for a single phase (liquid) flow. Second, the cavitation model is turned on and the steady equations are solved, with the vapour volume fraction, and therefore the liquid-vapour mixture density, as an additional unknown.

\section{Numerical approach, computational domain and boundary conditions}

We investigate the cavitational behaviour of a large storage pump with two identical stages. The two stages are coupled in-line in order to obtain a high pumping head. We study only the flow in the impeller of the first stage without taking into consideration the collector and the inlet section. The storage pump is presented in Fig. 1:

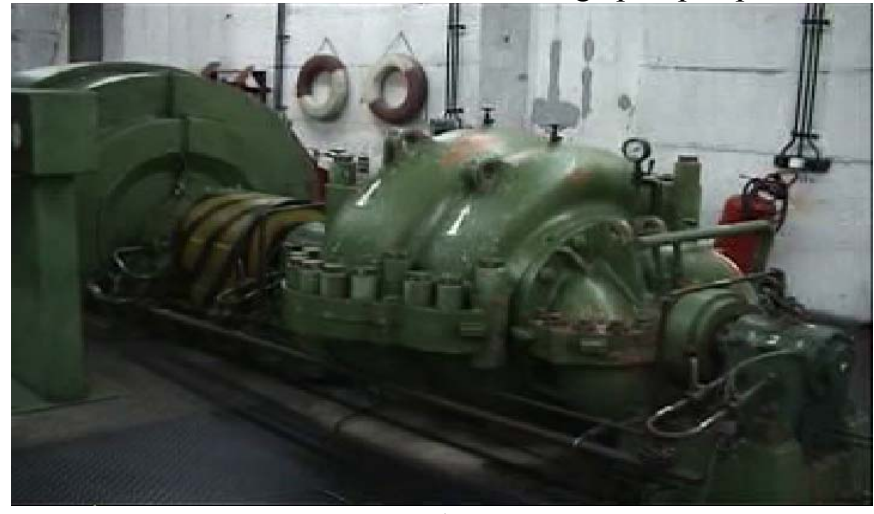

a)

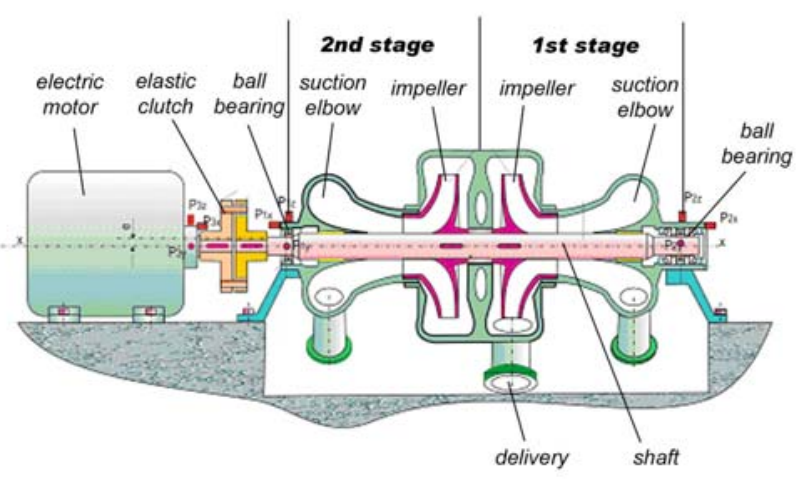

b)

Fig. 1 a) The ensemble of storage pump with the electrical motor

b) Cross section through the storage pump

The specifications of the impeller of the first stage of the storage pump, according to the manufacturer, are given in Tab. 1.

Table 1 Impeller geometry

\begin{tabular}{c|c|c|c}
\hline \hline & Symbol & Value & Unit \\
\hline Rotating speed & $\mathrm{n}$ & 1500 & $\mathrm{rpm}$ \\
\hline Design flow rate & $\mathrm{Q}_{\text {bep }}$ & 1 & $\mathrm{~m}^{3} / \mathrm{s}$ \\
\hline Head & $\mathrm{H}_{\text {bep }}$ & 159.5 & $\mathrm{~m}$ \\
\hline Number of blades & $\mathrm{n}_{\mathrm{p}}$ & 5 & - \\
\hline Inlet diameter & $\mathrm{D}_{1}$ & 380 & $\mathrm{~mm}$ \\
\hline Outlet diameter & $\mathrm{D}_{2}$ & 840 & $\mathrm{~mm}$ \\
\hline Inlet height & $\mathrm{b}_{1}$ & 140 & $\mathrm{~mm}$ \\
\hline Outlet height & $\mathrm{b}_{2}$ & 80 & $\mathrm{~mm}$ \\
\hline
\end{tabular}

To simulate the cavitating flow the numerical code FLUENT [6] was used. The code uses a control volume-based technique to convert the governing equations in algebraic equations that can be solved numerically. This control volume technique consists of integrating the governing equations at each control volume, yielding discrete equations that conserve each quantity on a controlvolume basis. The governing integral equations for the conservation of mass and momentum, and (when appropriate) other scalars, such as turbulence, are solved sequentially. Being the governing equations non-linear (and coupled), several iterations of the solution loop must be performed before a converged solution is obtained. The flow solution procedure is the SIMPLE routine [6]. This solution method is designed for incompressible flows, thus being semi-implicit. The full Navier-Stokes equations are solved. The flow was assumed to be steady, and isothermal. In these calculations turbulence effects were considered using turbulence models, as the k- $\varepsilon$ RNG models, with the modification of the turbulent viscosity for multiphase flow. To model the flow close to 
the wall, enhanced wall functions approach has been used to model the near-wall region (i.e., laminar sub layer, buffer region, and fully-turbulent outer region). For this model, the used numerical scheme of the flow equations was the segregated implicit solver. The SIMPLE scheme was employed for pressure-velocity coupling, first-order up-wind for the momentum equations and for other transport equations (e.g. vapour transport and turbulence modelling equations). Computational domain is meshed using the GAMBIT pre-processor [6].

The computational domain includes the impeller of the first stage of the storage pump. For the numerical investigation only one inter-blade channel is used because of the symmetry of the geometry, Fig. 2a.

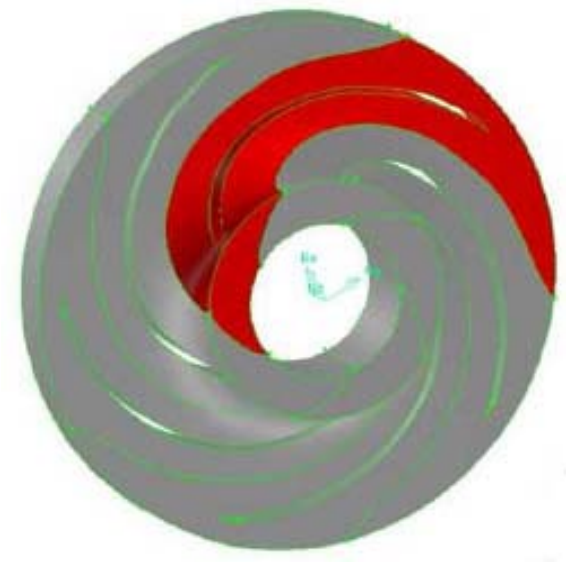

a)

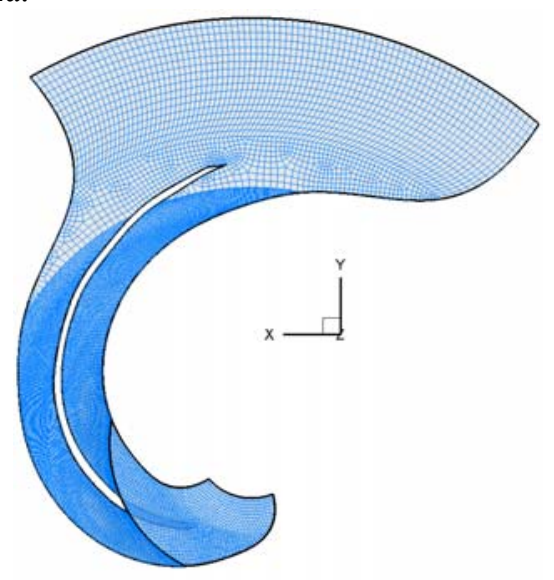

b)

Fig. 2 a) Impeller of the storage pump with highlighted inter blade channel

b) Mesh generated on the 3D computational domain of the inter-blade channel

The inter-blade channel domain is meshed with 322726 cells using a structured mesh, see Fig. $2 \mathrm{~b}$.

On the inlet surface of the impeller, for the liquid phase, a constant velocity field was imposed normal on the surface. The velocity magnitude is computed using the flow rate of the operating point:

$$
v_{L_{-} I N}=\frac{Q}{S_{I N}}
$$

On the outlet surface a constant value of the pressure is imposed. Then, the pressure is lowered slowly down to the value corresponding to the desired cavitation number $\sigma$ defined as:

$$
\sigma=\frac{p_{m_{-} I N}-p_{v a p}}{\rho_{m} g H}
$$

Vapour appears during the pressure decrease. After obtaining a steady single phase (liquid) flow solution, FLUENT 6.3 code allows turning on the cavitation model. As a consequence, vapour formation is enabled where the absolute pressure is smaller than the vaporization pressure, $p_{\text {vap }}$. In order to obtain correct results the operating pressure, $p_{\text {op }}$, must be set to zero (by default is equal with the atmospheric pressure), therefore the gauge pressure, $p_{\text {gauge, }}$, will be equal with the absolute pressure, $p_{a b s}$ :

$$
p_{a b s}=p_{o p}+p_{\text {gauge }}
$$

This setting is important for obtaining only positive absolute pressure values.

On the periodic surfaces of the impeller the periodicity of the velocity, pressure and turbulence parameters were imposed:

$$
\begin{aligned}
& p(r, \theta, z)=p\left(r, \theta+\frac{2 \pi}{n_{p}}, z\right) \\
& \vec{v}(r, \theta, z)=\vec{v}\left(r, \theta+\frac{2 \pi}{n_{p}}, z\right) \\
& k(r, \theta, z)=k\left(r, \theta+\frac{2 \pi}{n_{p}}, z\right) \\
& \varepsilon(r, \theta, z)=\varepsilon\left(r, \theta+\frac{2 \pi}{n_{p}}, z\right)
\end{aligned}
$$

The remaining boundary conditions for the impeller domain correspond to zero relative velocity on the blade, crown and hub.

Figure 3 shows the 3D computational domain with boundary conditions corresponding to an inter-blade channel of the impeller. The computational domain is bounded upstream by an annular section (wrapped on the same annular surface as the suction outlet section, but different in angular extension) in order to impose the boundary conditions on the outlet section. 


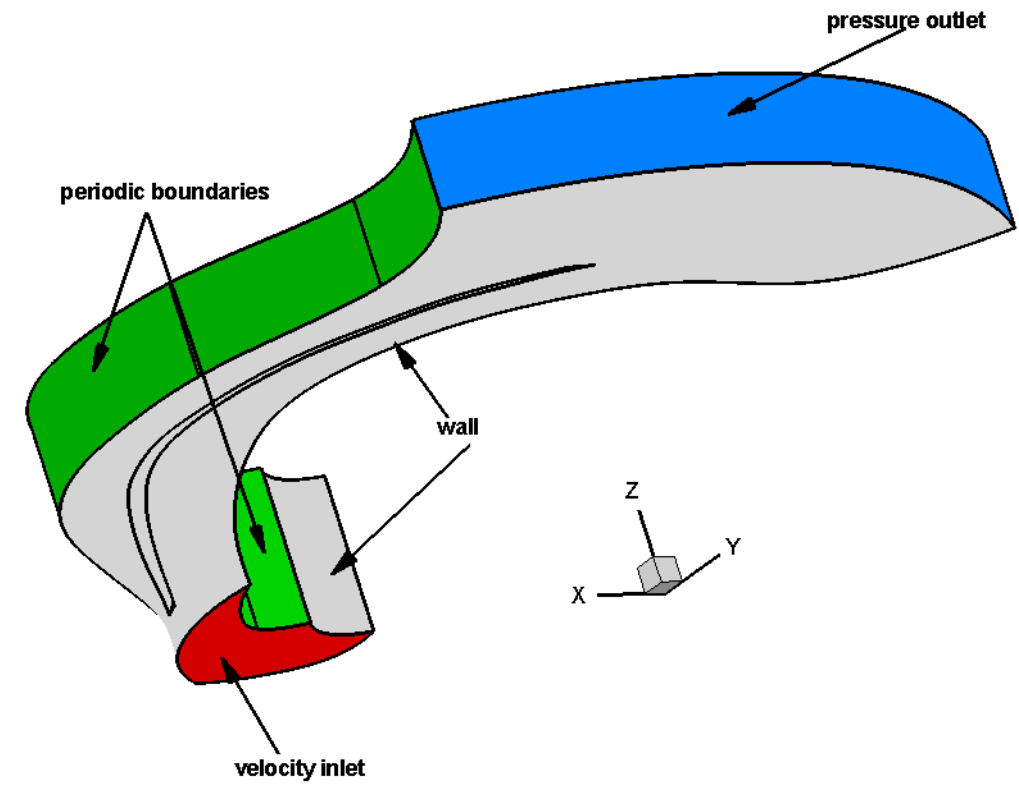

Fig. 3 Boundary conditions on the computational domain

We investigated the first stage of the storage pump at 5 operating points with the characteristics, according to the pump manufacturer, given in Tab. 2:

Table 2 Values of the main characteristics for the operating points

\begin{tabular}{c|c|c|c}
\hline \hline Operating points & $\begin{array}{c}n \\
{[\mathrm{rot} / \mathrm{min}]}\end{array}$ & $\begin{array}{c}Q \\
{\left[\mathrm{~m}^{3} / \mathrm{s}\right]}\end{array}$ & $\begin{array}{c}H \\
{[\mathrm{~m}]}\end{array}$ \\
\hline \multirow{5nn}{*}{ OP1 } & 0.8 & 175 \\
\cline { 1 - 2 } OP2 & \multirow{4}{*}{1500} & 0.9 & 169 \\
\cline { 4 - 4 } OP3 & 1.02 & 159.5 \\
\hline OP4 & 1.1 & 149 \\
\hline OP5 & 1.2 & 134.5 \\
\hline
\end{tabular}

\section{Numerical results}

In order to compute the pumping head from the data obtained from the numerical simulation of the multiphase turbulent flow in the impeller the following equation is used:

$$
H=\frac{p_{m_{-} O U T}-p_{m_{-} I N}}{\rho_{m} g}+\frac{v_{m_{-} \text {OUT }}^{2}-v_{m_{-} I N}^{2}}{2 g}+z_{\text {OUT }}-z_{I N}
$$

If the total pressure is given by:

$$
p_{m_{-} t o t}=p_{m}+\frac{\rho_{m} v_{m}^{2}}{2}
$$

and the difference between the inlet and outlet position is negligible then the pumping head has the following expression:

$$
H=\frac{p_{m_{-} t o t \_O U T}-p_{m_{-} t o t_{-} I N}}{\rho_{m} g}=\frac{\Delta p_{m_{-} t o t}}{\rho_{m} g}
$$

The suction head is determined with the equation:

$$
H_{s}=\frac{p_{\text {atm }}-p_{m_{-} t o t_{-} I N}}{\rho_{m} g}
$$

The cavitation number of the installation is given by:

$$
\sigma_{\text {inst }}=\frac{\frac{p_{\text {atm }}}{\rho_{m} g}-\frac{p_{\text {vap }}}{\rho_{m} g}-H_{s}}{H}
$$

The numerical evaluation of the vapour volume (the vapour includes both liquid vapour and non-condensable air) is obtained by integrating over the computational domain the vapour volume fraction, FLUENT [6]:

$$
V_{V}=\int_{V} \alpha_{V} d V
$$

The vapour volume fraction has values between 0 and 1 within each cell. As a result, when integrating the vapour volume 
fraction over the whole computational volume, only cells with non-zero $\alpha_{V}$ will have a contribution. This is the case in the region with low enough pressure for the noncondensable gas (air) to accumulate and for the liquid to turn into vapour. There is no distinct separation surface between the full vapour and full liquid regions. The mixture model assumes a continuous transition (with steep gradient, eventually) between the two phases, and it seems appropriate for practical applications where there is a bubble cloud instead a smooth sharp interface between the gas and liquid phases.

The connection between the relative volume of vapour, representing the ratio between the volume of vapour from the interblade channel and the volume of the inter-blade channel, and the flow rate is given by the following equation:

$$
\frac{V_{V}}{V}=A \cdot e^{B \cdot \sigma_{\text {inst }}}, \quad V=0.010417187 \mathrm{~m}^{3}
$$

where A, B are two coefficients specific for every operating point. According to our new approach, the interdependency between the relative volume of vapour and the flow rate can be represented using a semi-logarithmic plot, meaning that on the $0 \mathrm{Y}$ axis we represent $\ln \left(\frac{V_{V}}{V}\right)$, but the values on the axis of the plot are in fact the value of the $\frac{V_{V}}{V}$.

From the numerical simulation of the multiphase flow for the 5 operating points of the first stage of the storage pump we obtained the following results describing the variation of the relative volume of vapour as a function of the cavitation number of the installation for a single inter-blade channel, Fig. 4, Stuparu et al. [13]:

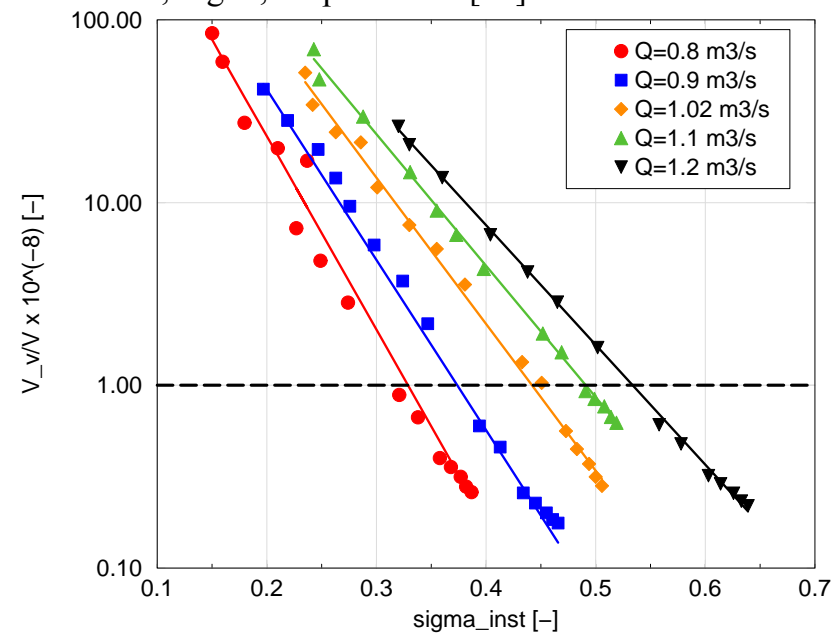

Fig. 4 Variation of the volume of vapour against the cavitation number for the investigated operating points

In order to determine the cavitation inception coefficient, it was considered that the relative volume of vapour of $10^{-8}$ corresponds to the volume of the first bubble, so the cavitation coefficient corresponding to this value of the relative volume of vapour represents the cavitation inception coefficient. The advantage of this method is that the value of the relative volume of vapour could be considered different of $10^{-8}$ if a more accurate result is needed and the corresponding value of the cavitation inception coefficient can be easily determined from the curves represented in Fig. 4. It is our opinion that by adopting a clear quantitative criterion instead of the rather qualitative (the "first bubble") or mesh dependent (void present in at least one cell) approaches would be preferable. Moreover, the exponential variation of the relative volume of vapour versus the cavitation number allows us to consider a fit that accounts for a whole range of $\sigma_{\text {inst }}$ values, instead of looking only for a particular (and rather sensitive) value of $\sigma_{i}$.

This new approach allows us also to compare the cavitational behaviour of the same turbo machine operating at variable discharge or of different turbomachines operating at the same discharge by comparing the slope of the curves represented in Fig. 4 . A higher value of the slope of the curve represents a higher cavitation sensitivity of the turbo machine, while a smaller value of the slope of the curve predicts lower cavitation sensitivity. So, if one has obtained the curves describing the cavitational behaviour of a hydraulic turbomachines, not just a pump, it can easily determine, by comparison of these curves, which turbomachines has a better cavitational behaviour.

For the centrifugal pump investigated it results from Fig. 4 that the slope of the curve increases with the decrease of the flow rate. That shows that the cavitational behaviour of the investigated pump is getting worse while the flow rate decreases.

From Fig. 4 it results the following values for the cavitation inception coefficient presented in Tab. 3 and Fig. 5:

Table 3 Values of the cavitation inception coefficient for the operating points

\begin{tabular}{c|c|c}
\hline \hline Operating points & $\begin{array}{c}Q \\
{\left[\mathrm{~m}^{3} / \mathrm{s}\right]}\end{array}$ & $\begin{array}{c}\sigma_{\mathrm{i}} \\
{[-]}\end{array}$ \\
\hline OP1 & 0.8 & 0.32 \\
\hline OP2 & 0.9 & 0.38 \\
\hline OP3 & 1.02 & 0.44 \\
\hline OP4 & 1.1 & 0.49 \\
\hline OP5 & 1.2 & 0.53 \\
\hline
\end{tabular}




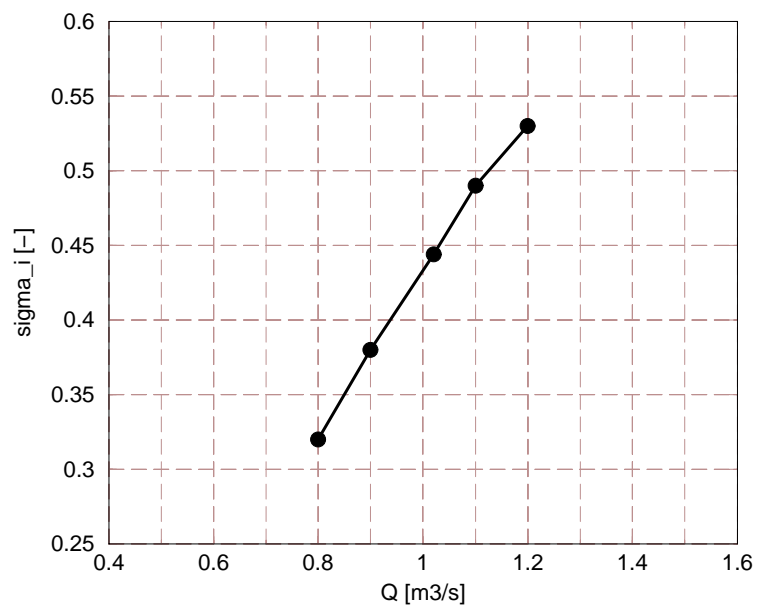

Fig. 5 Evolution of the cavitation inception coefficient as a function of flow rate

From Fig. 5 it results that the value of the cavitation inception coefficient increases with the value of the flow rate.

Dividing the pumping head with the value of the pumping head corresponding to the zero cavitation situation and representing the result as a function of $\frac{V_{V}}{V}$ for all the investigated operating points in a semi-logarithmic plot, where the $0 \mathrm{X}$ axis is the logarithmic one, Fig. 6, the following equation was obtained for the curve which fits best the data obtained, Eq. 42:

$$
\frac{H}{H_{0}}=1-0.0100374 \cdot\left(\frac{V_{V}}{V_{L}}-0.176596\right)^{0.489854}
$$

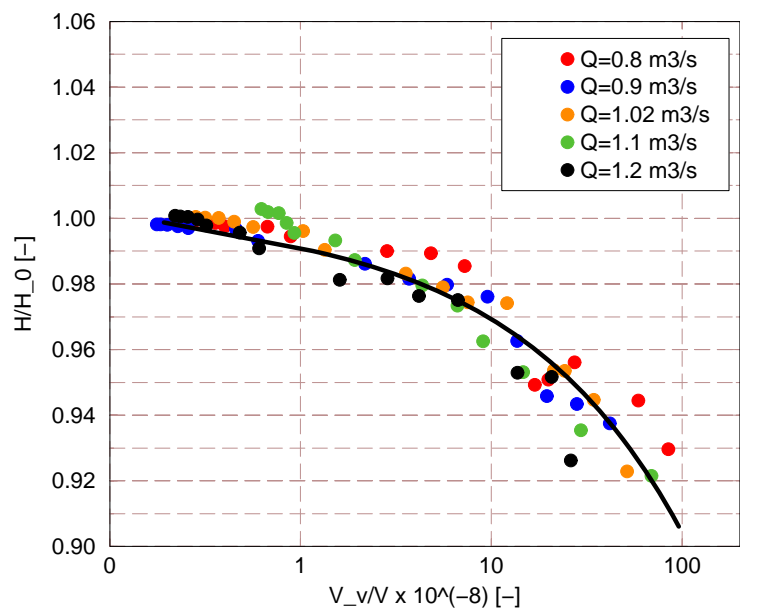

Fig. 6 Variation of the pumping head ratio against the volume of vapour for the investigated operating points

Figure 6, representing the mastre curve of relative head drop as the dimensionless vapour volume increses, and Equation 42 underlines the fact that the pumping head drop is dependent and caused mainly by the increase of the volume of vapour inside the inter-blade channel of the turbo machine.

Taking into account Eq. 41 and Eq. 42, the expression of the fiting curve for the values of the pumping head as a function of the cavitation number of the instalation can be easily obtained:

$$
H=H_{0} \cdot\left[1-0.0100374 \cdot\left(A \cdot e^{B \cdot \sigma_{\text {inst }}}-0.176596\right)^{0.489854}\right]
$$

where the coefficients $A$ and $B$ have specific values for each operating point and they are determined from Fig. 4 and presented in Tab. 4:

Table 4 Values of the curve coefficients for the operating points

\begin{tabular}{c|c|c|c}
\hline \hline Operating points & $\begin{array}{c}Q \\
{\left[\mathrm{~m}^{3} / \mathrm{s}\right]}\end{array}$ & $A$ & $B$ \\
\hline OP1 & 0.8 & 3006.31 & -24.3447 \\
\hline OP2 & 0.9 & 3049.22 & -21.4614 \\
\hline OP3 & 1.02 & 3473.95 & -18.4449 \\
\hline OP4 & 1.1 & 3415.98 & -16.5681 \\
\hline OP5 & 1.2 & 3126.79 & -15.0727 \\
\hline
\end{tabular}


Aplying Eq. 43 to the calculated data and taking into account the value of the coefficients from Tab. 4 the following graphical representation is obtained, Fig. 7:

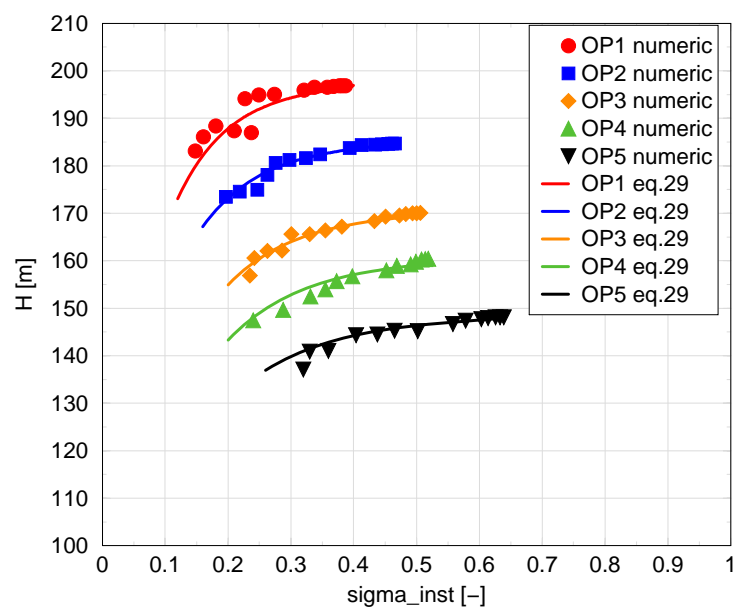

Fig. 7 Pumping head drop due to cavitation phenomena

Analysing Fig. 7 it can be observed that the curves obtained from Eq. 43 overlap very well the results obtained from postprocessing the data of the numerical simulation of the cavitating two phase flow. This indicates that Eq. 43 has the right expression.

\section{Conclusions}

In this paper we use standard software with an implemented cavitation model, in order to obtain the necessary data for the construction of the cavitation curves witch allows use to determine the cavitational behaviour of a centrifugal storage pump.

A new and robust method for the determination for the cavitation inception coefficient and cavitational behaviour of a centrifugal pump is presented. By using this method one can obtain with good precision the value of the cavitation inception coefficient, giving a desired value to the relative volume of vapour corresponding to the volume of the first bubble of vapour. This approach also allows someone who uses it to determine and compare the sensitivity to cavitation for the same turbo machine operating at different flow rates or of different turbomachines operating in the same range of flow rates. The sensitivity to cavitation of a turbo machine is determined from analysing the slope of the curve obtained from Eq. 41 and represented in a semilogarithmic plot. A higher value of the slope indicates a higher sensitivity to cavitation because of the quick increase of the volume of vapour.

We apply this new method for analysing the cavitational behaviour of the impeller from the first stage of a large centrifugal storage pump. It results that the value of the cavitation incipience coefficient increases while the flow rate increases, while the cavitation sensitivity of the centrifugal pump is getting higher with the decreasing of the flow rate.

The analyse of the results of the numerical investigation of the multiphase flow inside the storage pump underlines the fact that the pumping head drops due to the development of cavitation phenomena, while the level of the water from the suction lake drops, for all five operating points. It is obviously that, while the cavern filled with vapour grows, the perturbation of the flow on the suction side of the blade is more accentuated, leading to the detachment of the flow from the blade, the decreasing of the deviation realised by the impeller blades, and consequently to the pumping head drop. The connection between the pumping head drop and the increase of the volume of vapour is given by Eq. 42 . With the help of Eq. 41 and Eq. 43 the connection between pumping head drop and cavitation coefficient of the installation is realised.

The cavitation phenomenon appears due to the unfavourable hydrodynamics generated by the geometric shape of the impeller blades, the leading edge of the blades being very sharp. In order to avoid the appearance of cavitation it is necessary to redesign the impeller with modern design methods and software.

\section{Acknowledgements}

Dr. Sebastian Muntean was supported by the Romanian Academy program "Hydrodynamics Optimization and Flow Control of the Hydraulic Turbomachinery in order to improve the Energetic and Cavitational Performances".

\section{Nomenclature}

$\begin{array}{ll}\vec{a} & \text { acceleration }\left[\mathrm{m} / \mathrm{s}^{2}\right] \\ b_{1} & \text { inlet height of the impeller }[\mathrm{mm}] \\ b_{2} & \text { outlet height of the impeller [mm] } \\ c_{d r a g} & \text { drag function [-] } \\ C_{e} & \text { empirical constant [-] } \\ C_{c} & \text { empirical constant [-] } \\ d_{V} & \text { diameter of the bubble for the vapour phase }[\mathrm{m}] \\ D_{1} & \text { inlet diameter of the impeller [mm] } \\ D_{2} & \text { outlet diameter of the impeller [-] }\end{array}$

$\begin{array}{ll}R_{e} & \text { vapour generation rate term [-] } \\ R e & \text { Reynolds number [-] } \\ S_{I N} & \text { area of the inlet section of the impeller }[\mathrm{m} / \mathrm{s}] \\ t & \text { time [s] } \\ v_{c h} & \text { characteristic velocity }[\mathrm{m} / \mathrm{s}] \\ \vec{v}_{d r, V} & \text { drift velocity for vapour phase }[\mathrm{m} / \mathrm{s}] \\ \vec{v}_{m} & \text { velocity of the liquid-vapour mixture }[\mathrm{m} / \mathrm{s}] \\ v_{\mathrm{m} \text { IN }} & \text { inlet liquid-vapour mixture velocity }[\mathrm{m} / \mathrm{s}] \\ v_{\mathrm{m} \text { OUT }} & \text { velocity on outlet for liquid-vapour mixture }[\mathrm{m} / \mathrm{s}]\end{array}$




\begin{tabular}{|c|c|c|c|}
\hline$f_{g}$ & mass fraction of the noncondensable gases [-] & $\vec{v}_{L}$ & velocity of the liquid phase $[\mathrm{m} / \mathrm{s}]$ \\
\hline$f_{L}$ & mass fraction of the liquid [-] & $v_{\mathrm{L} \text { IN }}$ & velocity on inlet for liquid phase $[\mathrm{m} / \mathrm{s}]$ \\
\hline$f_{V}$ & mass fraction of the vapour [-] & $\vec{v}_{V L}$ & slip velocity $[\mathrm{m} / \mathrm{s}]$ \\
\hline$\vec{F}$ & body force $[\mathrm{N}]$ & $\vec{v}_{V}$ & velocity of the vapour phase $[\mathrm{m} / \mathrm{s}]$ \\
\hline$\vec{g}$ & gravitational acceleration $\left[\mathrm{m} / \mathrm{s}^{2}\right]$ & $V_{V}$ & volume of vapour $\left[\mathrm{cm}^{3}\right]$ \\
\hline$H$ & pumping head $[\mathrm{J} / \mathrm{N}]$ & $V$ & volume of the inter-blade channel $\left[\mathrm{m}^{3}\right]$ \\
\hline$H_{0}$ & pumping head without cavitation $[\mathrm{J} / \mathrm{N}]$ & $z$ & axial coordinate $[\mathrm{m}]$ \\
\hline$H_{s}$ & suction head $[\mathrm{J} / \mathrm{N}]$ & $z_{I N}$ & inlet position $[\mathrm{m}]$ \\
\hline$k$ & turbulent kinetic energy $\left[\mathrm{m}^{2} / \mathrm{s}^{2}\right]$ & $z_{\text {OUT }}$ & outlet position [m] \\
\hline$\dot{m}_{L V}$ & rate of liquid-vapour mass transfer $\left[\mathrm{kg} /\left(\mathrm{s} \mathrm{m}^{-3}\right)\right]$ & $\alpha_{\mathrm{g}}$ & noncondensable gases volume fraction [-] \\
\hline$\dot{m}_{V L}$ & rate of vapour-liquid mass transfer $\left[\mathrm{kg} /\left(\mathrm{s}^{-\mathrm{m}^{-3}}\right)\right]$ & $\alpha_{\mathrm{L}}$ & liquid volume fraction [-] \\
\hline$n$ & rotational speed [rot/min] & $\alpha_{V}$ & vapour volume fraction [-] \\
\hline$n_{p}$ & number of blades $[-]$ & $\varepsilon$ & dissipation rate $\left[\mathrm{m}^{2} / \mathrm{s}^{2}\right]$ \\
\hline$p_{m}$ & liquid-vapour mixture pressure $[\mathrm{Pa}]$ & $\gamma$ & effective exchange coefficient [-] \\
\hline$p_{m}$ tot & liquid-vapour mixture total pressure $[\mathrm{Pa}]$ & $\mu_{m}$ & liquid-vapour mixture viscosity [ $\mathrm{kg} / \mathrm{m} . \mathrm{s}]$ \\
\hline$p_{a b s}$ & absolute pressure $[\mathrm{Pa}]$ & $\mu_{L}$ & liquid phase viscosity $[\mathrm{kg} / \mathrm{m} . \mathrm{s}]$ \\
\hline$p_{\text {atm }}$ & atmospheric pressure $[\mathrm{Pa}]$ & $\mu_{V}$ & vapour phase viscosity $[\mathrm{kg} / \mathrm{m} . \mathrm{s}]$ \\
\hline$p_{\text {gauge }}$ & gauge pressure $[\mathrm{Pa}]$ & $P_{g}$ & noncondensable gases density $\left[\mathrm{kg} / \mathrm{m}^{3}\right]$ \\
\hline$p_{o p}$ & operating pressure $[\mathrm{Pa}]$ & $\rho_{m}$ & liquid-vapour mixture density $\left[\mathrm{kg} / \mathrm{m}^{3}\right]$ \\
\hline$p_{m I N}$ & inlet liquid-vapour mixture pressure [Pa] & $\rho_{L}$ & liquid density $\left[\mathrm{kg} / \mathrm{m}^{3}\right]$ \\
\hline$p_{m}$ OUT & outlet liquid-vapour mixture pressure [Pa] & $\rho_{\mathrm{V}}$ & vapour density $\left[\mathrm{kg} / \mathrm{m}^{3}\right]$ \\
\hline$p_{m_{-} \text {tot_LN }}$ & inlet liquid-vapour mixture total pressure $[\mathrm{Pa}]$ & $\sigma$ & cavitation number [-] \\
\hline$p_{m_{-} \text {tot_out }}$ & outlet liquid-vapour mixture total pressure $[\mathrm{Pa}]$ & $\sigma_{d}$ & Prandtl dispersion coefficient [-] \\
\hline$p_{\text {sat }}$ & liquid saturation vapour pressure $[\mathrm{Pa}]$ & $\sigma_{\text {inst }}$ & cavitation number of the installation $[-]$ \\
\hline$p_{\text {turb }}$ & pressure fluctuation induced by turbulence $[\mathrm{Pa}]$ & $\sigma_{i}$ & incipience cavitation number [-] \\
\hline$p_{\text {vap }}$ & vaporization pressure $[\mathrm{Pa}]$ & $\sigma_{t}$ & surface tension coefficient of the liquid $[\mathrm{kg} / \mathrm{m}]$ \\
\hline$Q$ & flow rate $\left[\mathrm{m}^{3} / \mathrm{s}\right]$ & $\theta$ & angular coordinate [rad] \\
\hline$r$ & radial coordinate $[\mathrm{m}]$ & $\tau_{V}$ & bubble relaxation time $[\mathrm{s}]$ \\
\hline$R_{c}$ & condensation rate term [-] & & \\
\hline
\end{tabular}

\section{References}

[1] Anton, I., 1985, “Cavitation,” Romanian Academy Publishing House, Bucharest, Vol. 2 (in Romanian).

[2] Bernad, S., Resiga, R., Muntean, S., and Anton, I., 2007, "Cavitation Phenomena in Hydraulic Valves. Numerical Modelling," Proceeding of the Romanian Academy, Series A, Vol. 8, No. 2.

[3] Manninnen, M., Taivassalo, V., Kallio, S., 1996, "On the mixture model for multiphase flow," VTT Publications 288, Technical research centre of Finland.

[4] Li, S. C., 2000, "Cavitation of Hydraulic Machinery," Imperial College Press.

[5] Singhal, A.K., Li, H.Y., Athavale, M.M., Jiang, Y., 2001, "Mathematical basis and validation of the full cavitation model," ASME FEDSM'01, New Orleans, Louisiana.

[6] FLUENT 6.3 User's Guide, 2002, Fluent Incorporated.

[7] Susan-Resiga, R., Muntean, S., Bernad, S., and Anton, I., 2003, "Numerical investigation of 3D cavitating flow in Francis turbines," in Proceedings of the International Conference on Modelling Fluid Flow (CMFF03), Budapest, Hungary. Vol. 2, pp. 950-957.

[8] Hirschi, R., Dupont, P. H., Avellan, F., Favre, J.-N., Guelich, J.-F., and Parkinson, E., 1998, "Centrifugal pump performance drop due to leading edge cavitation: numerical predictions compared with model tests," Journal of Fluids Engineering, Vol. 120, No. 6, pp. 705-711.

[9] Pouffary, B., Patella, R.F., Rebound, J.-L., Lambert, P.-A., 2008, "Numerical Simulation of 3D Cavitating Flows: Analysis of Cavitation Head Drop in Turbomachinery," Journal of Fluids Engineering, Vol. 130, pp. 061301-1 - 061301-10.

[10] Gonzalo Flores, N., Goncalves, E., Fortes Patella, R., Rolland, J., and Rebattet, C., 2008, "Head Drop of a Spatial Turbopump Inducer," Journal of Fluids Engineering, Vol. 130, Paper 111301.

[11] Ait-Bouziad, Y., 2006, "Physical Modelling of Leading Edge Cavitation: Computational Methodologies and Application to Hydraulic Machinery," PhD Thesis, EPFL, Lausanne, Switzerland.

[12] Schiller, L., Naumann, Z., 1935, “Z. Ver. Deutsch. Ing.," pp. 77-318.

[13] Stuparu, A.C., Susan-Resiga, R., Muntean, S., Anton, L.E., 2010, "Numerical Investigation of the Cavitational Behaviour into a Storage Pump at Off Design Operating Points," in Proceedings of the 25th IAHR Symposium on Hydraulic Machines and Systems, IOP Conference Series: Earth and Environmental Science, Timisoara, Romania, Vol. 12, DOI: 10.1088/17426596/12/1/011003. 